\title{
PERFIL DA AUTOMEDICAÇÃO DOS ALUNOS DE UMA ESCOLA TÉCNICA DO SUL DE MINAS GERAIS
}

\author{
${ }^{1}$ Matheus Ferreira RIOS \\ ${ }^{2}$ Walnéia Aparecida de SOUZA \\ ${ }^{3}$ Vanessa Martins de Souza SIQUEIRA \\ ${ }^{4}$ Márcia Helena Miranda Cardoso PODESTÁ \\ ${ }^{5}$ Gabriel Generoso Peixoto de MELO \\ ${ }^{6}$ Alexandre Guimarães ZUBA \\ ${ }^{7}$ João Cláudio Freitas Soares MACHADO \\ ${ }^{8}$ Eric Batista FERREIRA
}

${ }_{1,2,4,6,7,8}$ Universidade Federal de Alfenas-MG.
${ }^{3}$ Centro Universitário de Caratinga-MG.
${ }^{5}$ Unidade de Atenção Primária à Saúde de Guanhães-MG.

Recebido em: 30/10/2013 - Aprovado em: 20/12/2013 - Disponibilizado em: 15/01/2014

RESUMO: A automedicação é caracterizada pela iniciativa de um indivíduo utilizar um ou mais medicamentos sem orientação médica, seja por vontade própria ou pela influência de outras pessoas, visando tratar enfermidades ou aliviar sintomas. Assim, o presente estudo apresentou como objetivo avaliar a prevalência de automedicação entre estudantes de um Curso Técnico da área de Saúde. Trata-se de um estudo descritivo, transversal e quantitativo, realizado com 98 estudantes em Boa Esperança-MG. Utilizou-se como instrumento um questionário semi-estruturado, contendo variáveis relacionadas à automedicação. Os dados foram avaliados pelo teste exato de Fisher e Mann-Whitney a 5\% de significância. Com relação aos resultados, verificou-se que 78,6\% dos alunos praticam automedicação, sendo esta prática mais realizada pelos estudantes do Curso de Farmácia (85,5\%). Os medicamentos mais consumidos foram os analgésicos $(54,1 \%)$ e os antigripais $(52 \%)$. Dentre os agentes viabilizadores da automedicação, os pais foram os principais responsáveis $(42,5 \%)$. Com relação à utilização de chás medicinais, os alunos do curso de Enfermagem $(58,1 \%)$ fizeram maior utilização dessa prática que os alunos de Farmácia $(43,6 \%$; $\mathrm{p}=0,2218)$, sendo os pais ou familiares $(37,8 \%)$ os principais responsáveis pela indicação. Concluindo, a automedicação é uma realidade entre os alunos do curso técnico em saúde. Portanto, devemos conscientizar que a automedicação é uma prática de difícil controle, contudo existem meios para minimizá-la. Assim, a mídia e outros meios de comunicação devem apresentar-se como aliados na conscientização popular de que os medicamentos não são substâncias inócuas e podem prod ' indesejáveis levando a intoxicações graves.

Palavras-chave: Automedicação. Medicamentos. Prevalência. Estudantes.

\begin{abstract}
Self-medication is characterized by the initiative of an individual using one or more drugs without medical prescription, either willingly or by the influence of others in order to treat disease or relieve symptoms. Thus, the present study aimed to assess the prevalence of self-medication among students of a technical course in the area of Health. This is a descriptive, transversal and quantitative conducted with 98 students in Boa Esperança, Minas Gerais. It was used as an instrument a semi-structured survey, containing variables related to self-medication. Data were analyzed by Fisher's exact test and Mann-Whitney test at 5\% significance. Regarding the results, it was found that $78.6 \%$ of students practice self-medication, this practice being carried out by most students of the Pharmacy Course (85.5\%). The most used drugs were analgesics (54.1\%) and cold medicine (52\%). Among the enabler agents of self-medication, parents were the primarily responsible $(42.5 \%)$. With regard to the use of medicinal teas, the students of Nursing $(58.1 \%)$ made greater use of the practice than the pharmacy students $(43.6 \%, \mathrm{p}=0.2218)$, with parents or family members $(37.8 \%)$ primarily responsible for the nomination. In conclusion, self-medication is a reality among technical health students. Therefore, we must raise awareness that self-medication is a practice difficult to control, however there are ways to minimize it. Thus, the media and other means of communication should present themselves as allies in popular awareness that drugs are not innocuous substances and can produce undesirable effects leading to serious poisoning.
\end{abstract}

Key-words: Self-medication, medicines, prevalence, students. 


\section{INTRODUÇÃO}

A automedicação é definida como o uso de medicamentos sem prescrição médica, sendo o próprio paciente quem decide qual é o medicamento a ser utilizado, com o objetivo de tratar ou aliviar sintomas, independentemente da prescrição de um profissional (1). Esse conceito mostra que a população sempre buscou nos medicamentos uma solução milagrosa e imediata para suas patologias, frustrações, insucessos e infelicidade, transcendendo a atividade terapêutica dos medicamentos para a salvação de seus problemas, contribuindo, assim, para o maior consumo e consequentemente maior uso irracional destes (2).

As formas de se automedicar são múltiplas, tais como adquirir o medicamento sem prescrição; compartilhar remédios com a família, com vizinhos ou com amigos; reutilizar antigas prescrições e descumprir orientação de profissionais prescritores; prolongar ou interromper precocemente a posologia e o período de tempo indicados na prescrição, comprometendo, portanto, a eficácia da terapêutica (3).

O risco dessa prática está relacionado com o grau de instrução, com o acesso ao sistema de saúde, com os fatores econômicos, políticos e culturais que contribuem para a disseminação da automedicação, tornando essa prática um problema de saúde pública no mundo (4).
Segundo a Organização Mundial de Saúde e o Ministério da Saúde, o mercado brasileiro dispõe de mais de 32 mil medicamentos. No entanto, sabe-se que, para tratar uma ampla gama de doenças, cerca de 420 produtos seriam suficientes (3).

No Brasil, ainda há vários fatores que agravam o problema da automedicação, pois cerca de oitenta milhões de pessoas praticam a automedicação; há uma má qualidade de oferta de medicamentos; não se cumpre a obrigatoriedade da prescrição médica para algumas classes de fármacos e há uma carência de informações e de orientação da população em geral, o que justifica a preocupação com essa prática (5).

Os brasileiros estão consumindo, em média, 11 caixas de remédio por ano, sendo 8 delas sem orientação médica. No Brasil, são comercializados quase 2 bilhões de caixas de medicamentos, o que o torna o quarto na lista dos países que mais consomem produtos farmacêuticos, precedido por Estados Unidos, França e Alemanha (6). Mas, nesses outros países, há um rígido controle das agências reguladoras e o crescente envolvimento dos farmacêuticos no desestímulo dessa prática tornam menos crítica a situação.

Segundo The World Health Organization (7), a automedicação orientada é uma forma de autocuidado à saúde. A presença de farmacêuticos qualificados nas farmácias para encorajar o uso racional de medicamentos, informar sobre os fármacos e as complicações 
que podem advir de seu uso indiscriminado e, se necessário, encaminhar as pessoas para atendimento médico quando possível, promovendo a automedicação responsável.

Outro fator importante seria promover educação para a saúde, com a finalidade de informar a população sobre os riscos de se automedicar e propiciar uma maior conscientização sobre o uso correto de medicamentos; impedir a propaganda de medicamentos pela mídia, pois as inovações tecnológicas produzidas pelas indústrias farmacêuticas favorecem um aumento do consumo de medicamentos. Além disso, o aumento dos riscos de se intoxicar pela automedicação, tanto em países desenvolvidos como em desenvolvimento, resulta em um grave problema de Saúde Pública (8).

Tendo em vista que a automedicação é uma prática frequente, surge a necessidade de conhecer a prevalência dessa prática em nossa população que está se capacitando para trabalhar na área de saúde. Assim, esse estudo apresentou como objetivo a mensuração da prática de automedicação entre estudantes de uma Escola Técnica de Farmácia e Enfermagem da Cidade de Boa Esperança MG.

\section{MATERIAL E MÉTODOS}

Trata-se de um estudo transversal, de abordagem quantitativa, desenvolvido em uma Escola de Ensino Técnico na cidade de Boa Esperança, MG.

$\mathrm{O}$ inquérito foi realizado em caráter universal, isto é, com todos os alunos que ingressaram no segundo semestre de 2010, perfazendo um total de 43 alunos do Curso técnico de Enfermagem e 55 alunos do Curso Técnico de Farmácia, cujas idades variaram de 15 a 29 anos. O trabalho foi aprovado pelo Comitê de Ética em Pesquisa da Universidade Federal de Alfenas-MG, conforme protocolo $n^{\circ} 043 / 2010$.

Foi utilizado um instrumento estruturado e padronizado para a coleta dos dados. A aplicação do mesmo ocorreu em sala de aula, de forma simultânea, após detalhada explanação dos objetivos, tornando claro o anonimato dos alunos, bem como a exigência da leitura e a assinatura do Termo de Consentimento Livre e Esclarecido.

Por meio de um formulário, foram coletadas informações sociodemográficas como idade, gênero e o curso. Também foram avaliados o hábito de automedicar-se; se os medicamentos foram obtidos por orientação de farmacêuticos ou por indicação de terceiros (leigos ou não) ou o uso de medicamentos por conta própria. Também foi avaliada a procura ou não de orientação médica, assim como a presença de sintomas nos últimos 2 meses. Os resultados foram expressos pela média ( \pm desvio padrão). Quanto à estatística, foi analisada a associação da interferência do curso com o 
gênero, com a faixa etária, com a automedicação, com a indicação de medicamentos para outras pessoas, com a indicação para utilizar medicamentos e com a utilização de bebida alcoólica com medicamentos, pelo teste exato de Fisher e Mann-Whitney a 5\% de significância. Para a análise foi utilizado o software R (9).

\section{RESULTADOS}

Com relação ao perfil sociodemográfico dos entrevistados, verificou-se que o maior número de participantes foi do sexo feminino $(86,1 \%)$ no curso de enfermagem e no de farmácia, houve predominância do masculino (54,5\%). Mas, no geral, prevaleceu o sexo feminino $(63,3 \%)$. Já em relação ao grupo etário, houve maior número de participantes entre 20 e 22 anos (57,0\%). O estado civil predominante, em mais de $90 \%$ dos casos, foi o solteiro. A maioria $(85 \%)$ recebia um salário mínimo.

Em relação ao perfil de automedicação, 77 alunos $(78,6 \%)$ realizam essa prática. $\mathrm{Na}$ comparação da automedicação entre os cursos, essa prática foi maior entre os alunos do curso de farmácia (85,5\%); mas quando comparados os dois cursos, não houve diferença $(\mathrm{p}=0,1432)$. Os resultados encontram-se na Tabela 1.

Ao analisar a automedicação por faixa etária, os alunos de 15 a 18 anos corresponderam a $53,2 \%$ e os de 19 a 29 , a $46,8 \%$, apresentando diferença estatística significativa $(p=0,0148)$.

Quando analisados os hábitos dos alunos em indicar medicamentos para outras pessoas, verificamos que os do curso de enfermagem $(62,8 \%)$ realizaram essa prática em maior proporção que os de farmácia $(49,1 \%)$. Mas, quando comparados estatisticamente, não houve diferença ( $p=0,2208$ ). Os resultados estão apresentados na Figura 1.

Com relação aos motivos que levaram os alunos a utilizarem medicamentos nos últimos dois meses que antecederam a coleta de dados, verificou-se que a dor foi o motivo mais citado $(54,1 \%)$, especialmente cefaleia, dores musculares e dismenorreia, seguido de resfriados (52\%). Assim, os medicamentos mais utilizados sem prescrição foram os analgésicos e antigripais. Quando comparados em relação aos cursos, os alunos de farmácia

Tabela 1- Utilização de medicamentos pelos alunos dos Cursos Técnicos de Enfermagem e Farmácia da Escola de Boa Esperança-MG.

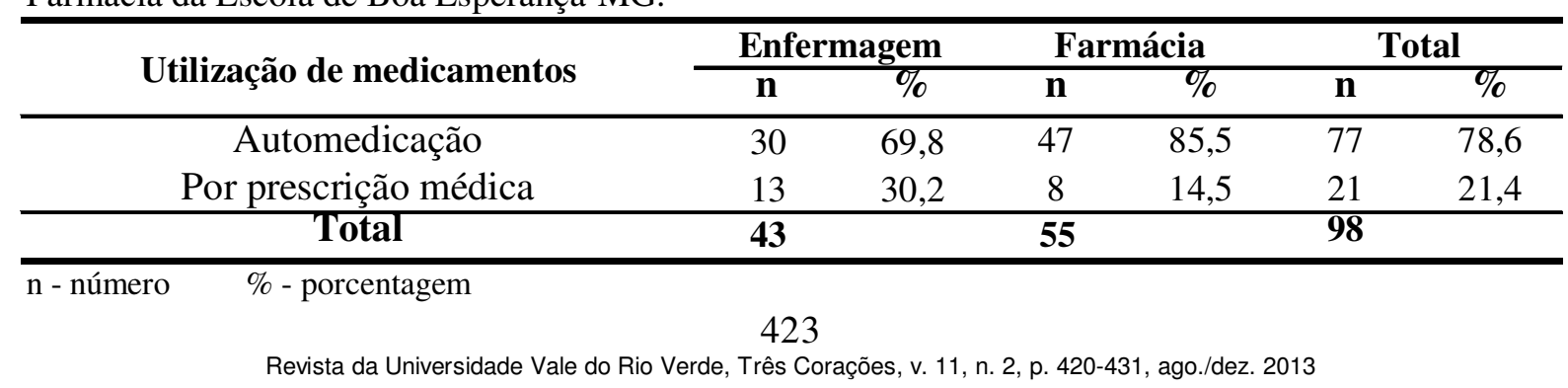




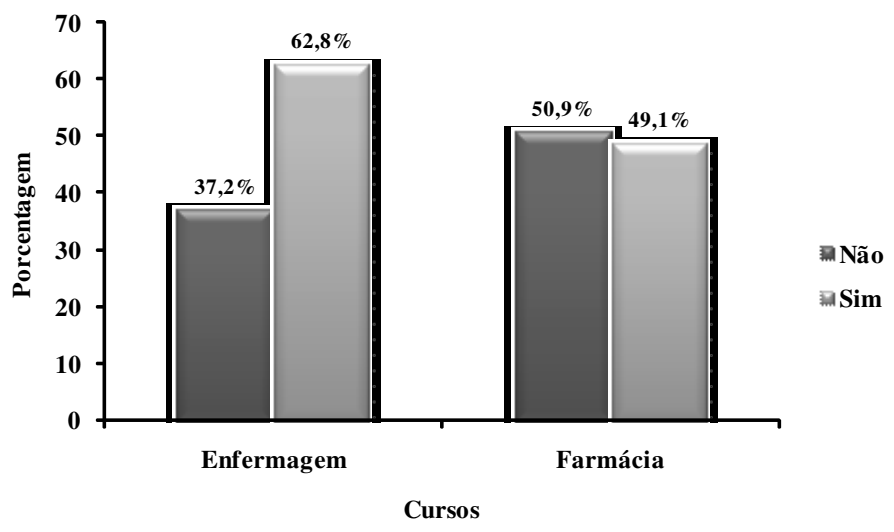

Figura 1- Distribuição percentual dos alunos dos Cursos Técnicos de Enfermagem e Farmácia da Escola de Boa Esperança-MG, que indicaram medicamentos para outras pessoas.

utilizaram $54,1 \%$ e os de enfermagem, $51,2 \%$

$(\mathrm{p}=0,681)$ dos analgésicos. Já em relação aos antigripais, os alunos de farmácia utilizaram $52 \%$ e os de enfermagem, $51,2 \%(\mathrm{p}=0,667)$.

Os resultados estão demonstrados na

Tabela 2.
Em relação ao sexo, os tipos de medicamentos mais utilizados pelas mulheres foram os analgésicos $(65,5 \%)$ e pelos homens $(62,9 \%)$, os antigripais, mas sem diferença estatística $(\mathrm{p}=0,865)$.

Tabela 2- Classes farmacológicas mais utilizadas na forma de automedicação entre os alunos dos Cursos Técnicos de Enfermagem e Farmácia da Escola de Boa Esperança-MG.

\begin{tabular}{lcccccc}
\hline \multirow{2}{*}{ Classes Farmacológicas } & \multicolumn{2}{c}{ Enfermagem } & \multicolumn{2}{c}{ Farmácia } & \multicolumn{2}{c}{ Total } \\
\cline { 2 - 7 } & $\mathbf{n}$ & $\mathbf{\%}$ & $\mathbf{n}$ & $\mathbf{\%}$ & $\mathbf{n}$ & $\mathbf{\%}$ \\
\hline Analgésicos & 22 & 51,2 & 31 & 56,4 & 53 & 54,1 \\
Antigripais em geral & 22 & 51,2 & 29 & 53 & 51 & 52 \\
Anti-inflamatórios/Antibióticos & 9 & 20,9 & 19 & 34,5 & 18 & 28,6 \\
Anticoncepcionais & 3 & 7 & 6 & 11 & 9 & 9,2 \\
Antiácidos e digestórios & 2 & 5 & 3 & 5,5 & 5 & 5,1 \\
Antidepressivos & 1 & 2,3 & 6 & 11 & 7 & 7,1 \\
Anorexígenos/laxantes & 1 & 2,3 & 1 & 2 & 2 & 2 \\
\hline
\end{tabular}

Os valores foram calculados em relação ao número de alunos de enfermagem (n=43) e farmácia (n=55).

Tabela 3 - Agentes viabilizadores responsáveis pela automedicação dos alunos dos Cursos Técnicos de Enfermagem e Farmácia da Escola de Boa Esperança-MG.

\begin{tabular}{lcccccc}
\hline \multirow{2}{*}{ Agentes Viabilizadores } & \multicolumn{2}{c}{ Enfermagem } & \multicolumn{2}{c}{ Farmácia } & \multicolumn{2}{c}{ Total } \\
\cline { 2 - 7 } & $\mathbf{n}$ & $\mathbf{\%}$ & $\mathbf{n}$ & $\mathbf{\%}$ & $\mathbf{n}$ & $\mathbf{\%}$ \\
\hline Pais ou responsáveis & 10 & 22,5 & 35 & $57,4^{*}$ & 45 & 42,5 \\
Amigos & 4 & 8,9 & 2 & 3,3 & 6 & 5,7 \\
Namorado ou esposo & 4 & 8,9 & 0 & 0 & 4 & 3,8 \\
Balconista da farmácia & 10 & 22,5 & 10 & 16,4 & 20 & 18,9 \\
Meios de comunicação & 2 & 4,4 & 4 & 6,6 & 6 & 5,7 \\
Por conta própria & 15 & 33,3 & 10 & 10,4 & 25 & 23,6 \\
\hline Total & $\mathbf{4 5}$ & & $\mathbf{6 1}$ & & $\mathbf{1 0 6}$ \\
\hline
\end{tabular}

$\mathrm{N}^{\circ}$ - número $\%$ - porcentagem $\quad * \mathrm{p}<0,05$ - considerado significativo. $\mathrm{O}$ aluno poderia responder mais de uma resposta. 
Já em relação à prevalência dos agentes viabilizadores da automedicação, verificou-se que os pais foram os principais responsáveis para os alunos do curso de farmácia $(57,4 \%)$ e para o curso de enfermagem, foi a utilização por conta própria $(22,2 \%)$. Os resultados estão demonstrados na Tabela 3.

A utilização de chás medicinais é uma prática muito utilizada pela população. Verificou-se que os alunos do curso de Enfermagem $(58,1 \%)$ fizeram maior utilização dessa prática que os alunos de Farmácia (43,6\%). Mas, quando comparados estatisticamente, não houve diferença significativa $(\mathrm{p}=0,2218)$.

\section{DISCUSSÃO}

No Brasil, onde o acesso à assistência médica pública é difícil e onde há uma grande parcela da sociedade na faixa da pobreza que não tem condições financeiras para pagar um plano de saúde ou que também não tem acesso ao Sistema Único de Saúde, a prática da automedicação torna-se bastante comum.

Mas, o fator financeiro não é o único responsável na prática da automedicação, pois fatores como escolaridade, gênero, classe social, acesso às informações a respeito dos medicamentos também entram nesse contexto (10).

Verificou-se que $78,6 \%$ dos entrevistados confirmaram a prática de automedicação nos últimos dois meses que antecederam à entrevista. $\mathrm{Na}$ análise da automedicação entre os cursos, $85,5 \%$ dos alunos eram do curso de farmácia. Mas, na comparação entre os dois cursos, não houve diferença estatística ( $p=0,1432)$. Isso pode ser justificado por serem cursos da área de saúde; sendo assim, apresentam conteúdos semelhantes, principalmente relacionados aos medicamentos.

Os resultados deste estudo mostram uma prevalência de automedicação elevada $(78,6 \%)$. Assim, este estudo apresenta-se semelhante a outros realizados com estudantes de graduação em algumas Universidades do Brasil, cujos resultados de automedicação em geral apresentaram elevados. Em uma Universidade de medicina em Santos, o índice de automedicação foi de 94,33\% (11); em acadêmicos de medicina da Universidade Federal de Juiz de Fora, o índice foi de 76,05\% (8); em estudos realizados com alunos de enfermagem da Universidade Paulista (UNIP), o índice foi de $65,17 \%$ (12). Outro estudo realizado com quatro cursos da área de saúde no Sul de Minas Gerais, o índice de automedicação foi de 93,11\%. Portanto, verificou-se que, na realidade, a automedicação é uma prática 
prevalente entre os acadêmicos da área de saúde.

Em nosso país, pelo menos $35 \%$ dos medicamentos são adquiridos por automedicação, ou seja, para cada dois medicamentos prescritos, pelo menos um é consumido sem orientação médica, sendo comum a reutilização de receitas (13).

Assim, com uma grande oferta de medicamentos e também com as propagandas da mídia, parece que os indivíduos precisam consumir algum tipo de medicamento para sentir-se bem e preencher seus vazios. Portanto, o medicamento se destaca como mais um bem de consumo acessível, como se não apresentasse riscos e pudesse ser consumido livremente (14).

Quando analisada a automedicação em relação ao sexo, o feminino apresentou maior prevalência no estudo geral $(63,3 \%)$. Essa prevalência do sexo feminino é explicada em alguns estudos, como o realizado por Bertoldi e Colaboradores (15), em Pelotas e por Vilarino e Colaboradores (16), em Santa Maria. Isso se deve à maior exposição das mulheres à medicalização em todas as fases de sua vida, maior procura por cuidados médicos e pela existência de campanhas educativas mais direcionadas a elas (14).

Não temos conhecimento do perfil de utilização de medicamentos entre alunos de outros cursos técnicos, mas vários estudos têm sido realizados na graduação com acadêmicos de diversas áreas. Muitos estudos publicados têm demonstrado que os acadêmicos da área de saúde se automedicam mais do que o de outras áreas (14). Isso pode estar relacionado com o maior conhecimento de sintomas das doenças, dos medicamentos e também dos seus efeitos pelos alunos dessa área (8).

Quando analisados os hábitos dos alunos de indicarem medicamentos para outras pessoas, verificamos que os do curso de enfermagem $(62,8 \%)$ indicaram maior número de vezes que os de farmácia $(49,1 \%)$, mas sem diferença significativa $(p=0,2208)$.

Estudos realizados com alunos de medicina da UNAERP apontam que 69,5\% dos alunos analisados responderam já terem indicado medicamentos para terceiros, uma prática considerada exercício ilegal da medicina (17). Em estudo realizado por Zafar e Colaboradores (18) em Karachi no Paquistão, com 572 universitários, aproximadamente $50 \%$ dos estudantes de medicina dessa universidade indicaram ou receitaram medicamentos informalmente, sem estar, obviamente, habilitados para prescrever

Com relação aos motivos que levaram os alunos a utilizarem medicamentos nos últimos dois meses que precederam a coleta de dados, verificou-se que a dor foi o motivo mais citado (51\%), especialmente cefaleia, dores musculares e dismenorreia, seguido de resfriados (48,8\%). Assim, os analgésicos $(51 \%)$ foram os medicamentos mais consumidos, fato que se mostrou semelhante 
a outros trabalhos desenvolvidos naquele país $(15,19)$.

Aspectos importantes em pesquisas sobre automedicação, independentemente da população em estudo, se referem às classes de medicamentos consumidos. Os antiinflamatórios, os analgésicos e os antitérmicos lideram a lista em diversos estudos realizados em diferentes populações $(8,19,20)$. A explicação para o maior uso desses medicamentos pode estar relacionada com a facilidade de aquisição e pela ideia de que se trata de produtos inofensivos, pois encontram-se exposto em caixas de supermercados e em mercearias, sem nenhuma restrição de venda (13).

Segundo Melo e Colaboradores (13), a venda de medicamentos anódinos em supermercados e em demais locais leigos pode engrossar as estatísticas de incidência de intoxicações medicamentosas e somente com uma fiscalização sanitária atuante, associada à educação popular, os perigos da automedicação poderiam tomar um rumo menos desastroso.

Com relação aos agentes viabilizadores da automedicação, constatou-se que houve em nosso estudo mais casos de indicação dos medicamentos por parte dos familiares $(42,5 \%)$, seguido do uso por conta própria $(23,6 \%)$ e da indicação por balconista $(18,9 \%)$. Diante desse fato, nota-se que os familiares exercem influência significativa no processo de cuidar e, assim, de se automedicar, sendo considerado por muitos estudos a principal influência para a ocorrência desse processo (21).

Estudos realizados por Garbossa e Colaboradores (22), demonstraram que os principais agentes viabilizadores da automedicação são os amigos, os parentes e os vizinhos. Loyola Filho e Colaboradores (23) comprovaram em seu estudo que compartilhar medicamentos com outros membros da família ou com outros moradores do domicílio e utilizar sobras de medicamentos (prescritos ou não) guardados no domicílio são duas modalidades de automedicação que podem ser favorecidas por um maior número de moradores do mesmo domicílio.

O subsistema familiar, que inclui o indivíduo e suas redes sociais, como familiares, amigos e vizinhos, é o mais importante e o mais utilizado do ponto de vista do itinerário terapêutico. É nele, primeiro, que se percebem e se experimentam os sintomas, rotulam-se e avalia-se a doença, sobre a qual se estabelece a condição aguda, crônica e sobre a qual se avalia o efeito do tratamento e da cura. Os primeiros cuidados são estabelecidos nesse setor os quais incluem, dentre outros, a alteração na alimentação, o repouso, o remédio caseiro, a automedicação, o suporte emocional e as práticas religiosas (24).

A pessoa e sua família utilizam crenças e valores sobre a doença, o que é 
parte da estrutura cognitiva da cultura popular, para validar a doença e o papel do doente, ou para desconsiderar sinais de doença por serem tratadas como naturais (24).

Assim, verificou-se que a confiança que é depositada nos familiares e nos amigos, a partir do vínculo existente, facilita o processo de automedicação. Portanto, a orientação da comunidade por meio da mídia e de outros veículos de comunicação é prérequisito para que seja promovida a conscientização sobre o uso de medicamentos, mediante indicação por profissionais de saúde que trabalham com o medicamento e que realmente tenham formação sobre o assunto.

A utilização de chás caseiros para tratar vários sintomas é uma realidade popular, promovida por crenças e tradições populares que se confundem com as propriedades curativas de muitas plantas silvestres (21). A população leiga em geral acredita que as plantas medicinais são inofensivas e elas continuam ocupando lugar de destaque no arsenal terapêutico. Segundo estimativas da Organização Mundial de Saúde (OMS), $80 \%$ da população mundial usa recursos da medicina popular para suprir as necessidades de assistência médica privada $(25,26)$. Assim, com base nessa estimativa, houve maior curiosidade na avaliação da utilização de chás medicinais para tratar sintoma ou doença.
Em relação ao índice de utilização de chás medicinais, verificou-se que os alunos do curso de Enfermagem $(58,1 \%)$ fizeram maior uso dessa prática que os alunos de Farmácia $(43,6 \%)$, mas sem apresentar diferença estatística significativa.

Com relação aos sintomas que desencadearam o uso de chás medicinais, a maior proporção $(34,7 \%)$ foi para gripe e resfriados, sendo os pais ou familiares $(37,8 \%)$ os principais responsáveis pela indicação. Segundo estudos realizados por Medeiros e Colaboradores (2004), a maior utilização também foi para o tratamento de gripe, seguido de bronquite. Novamente, verificou-se a influência dos familiares na indicação de produtos baseados no conhecimento popular. Verificou-se que esse tratamento é o primeiro de escolha e, somente após a não melhora dos sintomas, é que se faz uso dos medicamentos industrializados.

A investigação a respeito do uso de plantas medicinais também foi realizada, por ser uma prática muito utilizada em nosso país. A utilização de chás medicinais tem um lugar garantido no "folclore" brasileiro. Mas o efeito da maioria delas é desprovido de qualquer fundamentação científica (evidências) e a sua manipulação por leigos pode comprometer a qualidade dos chás e também promover interações com medicamentos industrializados e causar efeitos adversos graves (21). Assim, é preciso 
maior conscientização da popular em relação a essa prática.

Mesmo sendo o uso de plantas medicinais amplamente difundido no Brasil, ainda existe escassez de informações e a falta destas dá-se também entre indivíduos de nível superior, podendo gerar uso incorreto das mesmas, provocando o aparecimento de novas patologias e/ou o agravamento das já existentes. Os meios de comunicação já não são mais os grandes vilões do estímulo do uso das plantas medicinais, a cultura popular faz com que as indicações sejam transmitidas de pessoa para pessoa, atingindo também a população mais favorecida economicamente (25).

Portanto, verifica-se que as razões pelas quais as pessoas podem se automedicar são inúmeras. A propaganda desenfreada e massiva de determinados medicamentos contrasta com as tímidas campanhas que tentam esclarecer os perigos da automedicação. A dificuldade e o custo de se conseguir uma opinião médica; a limitação do poder prescritivo, restrito a poucos profissionais de saúde; o desespero e a angústia desencadeados por sintomas ou pela possibilidade de se adquirir uma doença; informações sobre medicamentos obtidos na internet ou em outros meios de comunicação; a falta de regulamentação e de fiscalização daqueles que vendem e a falta de programas educativos sobre os efeitos muitas vezes irreparáveis da automedicação, são alguns dos motivos que levam as pessoas a utilizarem medicamentos. (21).

$$
\text { A automedicação é um problema }
$$

universal, antigo e de grandes proporções, que permanecerá a nos assombrar com o aumento das internações e dos custos hospitalares devido às intoxicações e as consequências de sua utilização ao longo dos anos em órgãosalvo (fígado, rins, coração, etc.). A automedicação é considerada uma forma de não adesão às orientações médicas e de saúde. Assim, pela análise da literatura, não há como erradicar a automedicação, talvez pela própria condição humana de testar e de arriscar decisões. Há, contudo, meios para minimizá-la. Programas de orientação para profissionais de saúde, de farmacêuticos, de balconistas e da população em geral, além do estímulo a fiscalização apropriada, são fundamentais nessa situação.

\section{CONCLUSÕES}

Conclui-se que a automedicação se faz presente entre os alunos da Escola Técnica de Boa Esperança-MG.

Embora não se possa condenar totalmente essa prática, uma vez que existe uma grande parcela da população que tem dificuldade de acesso aos serviços de saúde, a automedicação torna-se um problema de grande proporção com várias questões inseridas e aparentemente como uma prática de difícil controle. 
Contudo, existem meios para minimizá-la e, para isso se tornar realidade, é preciso que os profissionais de saúde, em especial aqueles que trabalham diretamente com o medicamento, assumam o papel que lhes cabe na promoção da saúde. Faz-se necessária uma concentração de esforços destes profissionais na educação e na informação verdadeiramente comprometidas com o incentivo do uso racional dos medicamentos.

Assim, a mídia e outros meios de comunicação devem servir como aliados na conscientização popular de que os medicamentos são para uso individual e não devem ser repassados para outras pessoas, pois os mesmos não são substâncias inócuas e podem produzir efeitos indesejáveis levando a intoxicações graves.

\section{REFERÊNCIAS}

1. Berquó LS, Barros AJD, Lima RC, Bertoldi AD. Utilização de medicamentos para tratamento de infecções respiratórias na comunidade. Rev Saúde Públ 2004; 38(3):358-364.

2.Lefèvre F. A oferta e a procura de saúde através do medicamento: proposta de um campo de pesquisa. Rev Saúde Públ 1987; 21(1): 64-67.

3.Lopes NM. Automedicação: Algumas Reflexões Sociológicas. Sociologia, Problemas e Práticas 2001; 37:141-165.

4. Loyola filho AI, Uchôa E. Automedicação, motivações e características de sua prática. Rev. Méd Minas Gerais 2002; 12(4): 219-227.

5. Lima GB, De Araujo EJF, Sousa KMH, Benvido RF, Silva WC, Correa Jr. RAC,
Nunes LCC. Avaliação da utilização de medicamentos armazenados em domicílios por uma população atendida pelo PSF. Rev Bras Farm 2008; 89(2):146-149.

6. Cerqueira GS, Diniz MFFM, Lucena GT, Dantas AF, Lime GMB. Perfil da automedicação em acadêmicos de enfermagem na Cidade de João Pessoa. Rev Conceitos 2005; 11:123-126.

7.World Health Organization (WHO). The role of the pharmacist in self-medication and self-care. Genebra: WHO; 1998.

(WHO/DAP/09.13).

8.Neto JAC, Sirimarco MT, Choi CMK, Barreto AU, Souza JB. Automedicação entre Estudantes da Faculdade de Medicina da Universidade Federal de Juiz de Fora. $H U$ Rev Juiz de Fora 2006; 32(3):59-64.

9. R Development Core Team (2011). R: A language and environment for statistical computing. R Foundation for Statistical Computing, Vienna, Austria. ISBN 3-90005107-0. Disponível em < http://www.Rproject.org/ > Acesso em 2011.

10.Sousa, WO, Silva JL, Neto MS. A importância do profissional farmacêutico no combate à automedicação no Brasil. Rev Eletrônica Farm 2008;5(1):67-72.

11.Fonseca, FIRM et al. Frequência de automedicação entre acadêmicos de faculdade de medicina. Diagn Tratamento 2010; 15(2):53-57.

12.Santos B, Souza LG, Delgado NM, Torres WO. Incidência da automedicação em graduandos de Enfermagem. J Health Sci Inst 2012; 30(2):156-160.

13.Melo DO, Ribeiro E, Storpitis S. A importância e a história dos estudos de utilização de medicamentos. Rev Bras Ciênc Farm 2006; 42(4):475-485.

14.Aquino DS, Barros JAC, Silva MDP. A automedicação e os acadêmicos da área de saúde. Ciên Saúde Colet 2010; 15(5): 25332538.

15.Bertoldi AD, Barros AJD, Hallal PC, Lima RC. Utilização de medicamentos em adultos: prevalência e determinantes individuais. Rev Saúde Públ 2004; 38(2):228-238. 
16.Vilarino JF, Soares IC, Silveira CM, Rodel APP, Bortoli R, Lemos RR. Perfil da Automedicação em Município do Sul do Brasil. Rev Saúde Públ 1998; 32(1):43-49.

17.Silva RCG, Oliveira TM, Casimiro TS, Vieira KAM, Tardivo MT, Faria Jr M, Restini CBA. Automedicação em acadêmicos do curso de medicina. Medicina (Ribeirão Preto) 2012;45(1): 5-11.

18.Zafar SN, Syed R, Waqar S, Irani FA, Saleem S. Prescription of medicines by medical students of Karachi, Pakistan: A cross-sectional study. BMC Public Health 2008; 8:10-14

19.Arrais PSD, Coelho HLL, Batista MCDS, Carvalho ML, Righi RE, Arnau JM. Perfil da Automedicação no Brasil. Rev Saúde Públ 1997; 31(1):71-77.

20. Vosgerau MZS, Soares, DA, Souza RKT. Automedicação entre Adultos na Área de Abrangência de uma Unidade Saúde da Família. Lat Am J Pharm 2008; 27: 831-838.

21.Editorial. Automedicação. Rev Assoc Méd Bras 2001; 47(4):269-270.

22. Garbossa AF, Pegoraro F, Giacomelli GM, Nadal J,Furmann TPP, Thiesen LCT. Automedicação com analgésicos e antiinflamatórios na Cidade de Quedas do Iguaçu - PR. Rev Biol e Saúde da UNISEP 2007; 1(1).2: 9-15.

23.Loyola-Filho AI, Uchôa E, Guerra HL, Firmo JOA, Lima-Costa MF. Prevalência e fatores associados à automedicação: resultados do projeto Bambuí. Rev Saúde Públ 2002; 36(1):55-62.

24. Kleinman A. Concepts and a model for the comparison of medical system as cultural system. Soc Sci and Med 1978; 12:85-93.

25. Costa AFE, Frota JG, Lima MC, Moraes MO. Plantas medicinais utilizadas por pacientes atendidos nos ambulatórios do Hospital Universitário Walter Cantidio da Universidade Federal do Ceará. Pesq Méd Fortaleza 1998;1(2): 20-25.

26.Yunes RA, Pedrosa RC, Cechinel Filho V. Fármacos e fitoterápicos: a necessidade do desenvolvimento da indústria de fitoterápicos e fitofármacos no Brasil. Quím Nova 2001; 24(1):147-152. 